\section{A definition of entropy}

SIR-It is not easy to accept Charles Kittel's remarks (Nature 339, 170; 1989) concerning the definition of entropy. He objects that "no dictionary has advanced beyond the age of steam". While one may sympathize with his regrets that expositions of classical thermodynamics are often still written in a manner in keeping with the spirit of the industrial revolution, I reject his apodeictic definition of entropy as "the logarithm of the number of quantum states accessible to a system". First and foremost, entropy - like the second law of thermodynamics whence it arises - is about irreversibility. The logarithm of a number of quantum states, however, in no way suggests to the uninitiated any connection with this fundamental phenomenon; nor indeed is the irreversibility of natural processes easily established in reductionist theories. Moreover, Kittel's preferred definition

\section{Unstatistical bias}

SIR-Some of the British research councils calculate, for each university department, the proportion of students they support who complete their theses within four years. Those departments in which a certain percentage (currently the Economic and Social Research Council specify 35 per cent, Natural Environment Research Council 45 per cent, Science and Engineering Research Council 40 per cent) of the students fail to complete are 'blacklisted' and not given further studentships. This policy initially seems reasonable but may contain some major statistical injustices.

The issue can be considered by comparing two scenarios: (1) Some departments are lazy, inefficient and have low morale. Their students are slow in writing up and few complete within four years. Blacklisting would encourage such departments to

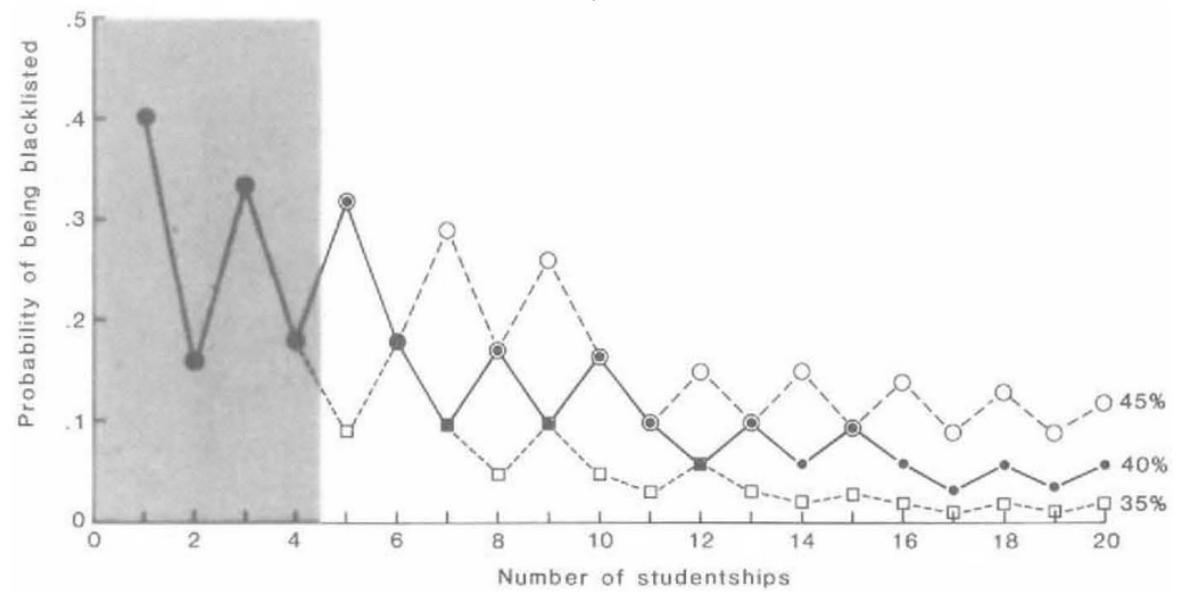

The probability of being blacklisted by chance for departments with different numbers of studentships. The three lines show blacklisting at three different thresholds. Departments with fewer than five studentships are excluded from blacklisting and are shaded. has here in the first instance a definition of empirical rather than of metrical entropy is of no concern in the present context.

H.A. BUCHDAHL

ological thermodynamics. Does this not imply that this powerful discipline is to be abandoned?

In short, I firmly believe that the ordinary, non-specialist dictionary should give pride of place to a contemporary phenomenological definition of entropy. Such a definition will reflect the existence of an ordering amongst the states of any adiabatically isolated system. On the lowest technical level it might be something like this: "the entropy of a system [in any given state] is a number [attached to that state] such that the relative adiabatic accessibility of any two states is governed by the difference between their entropies"; that is, an adiabatic transition from any initial state to any final state of lower entropy is impossible. (The assertion that such a labelling exists then amounts to a statement of the second law.) That one

mend their ways. (2) There is little real difference between departments. Due to chance events, there will be variation in the proportion of students completing their theses and some departments will have a higher success rate than others. With this scenario, blacklisting is demoralizing, unjust and shows an ignorance of statistics.

The probability of being blacklisted can be calculated from the binomial distribution, assuming that the average completion rate is 60 per cent. The relationship between this probability and department size is shown in the figure - most departments have fewer than ten studentships. This analysis shows that there are erratic differences between different sized departments and that there is considerably less risk for large departments.

WILLIAM J. SUTHERLAND

School of Biological Sciences,

University of East Anglia,

Norwich NR4 7TJ, UK thing as being wrong" could easily be

\section{Department of Physics}

and Theoretical Physics,

Australian National University,

GPO Box 4, Canberra,

ACT 2601, Australia

\section{What is truth?}

SIR-An unfortunate impression may have been given by your leading article Can heresy be real?'. Your somewhat ambiguous sentence "[the] standard complaint against the popperian view of science - that there is no such thing as truth - can also be turned inside out, to show that, in the fields in which hypotheses cannot yet be tested, there is no such understood to mean that, according to Popper, there is no such thing as truth. No-one who knows Popper's work could think so; but people sometimes do think so, as was shown by another recent article in Nature $^{2}$, where he was attacked, again ambiguously, as a "betrayer of the truth". The doctrine that truth does exist, and is the aim of science, has been held by Popper, following Tarski ${ }^{3}$, since 1935; yet it is hardly more popperian than the doctrine that the Earth is not flat. What is a distinctively popperian thesis is that we devise tests for hypotheses because they may be wrong (false), not to prove them right (true) and that by trying to eliminate error we may with luck (but never with certainty) latch on to some part of the objective and absolute truth.

Department of Philosophy,

University of Warwick,

Coventry CV4 7AL, UK

Tal 29, D-8000 Munich, FRG

DAVID MiLleR

1. Nature 340, 82 (1989).

2. Theocharis, t. \& Psimopoulos, M. Nature 329, 595-598 (1987).

3. Tarski, A. in Logic, Semantics, Metamathematics, 152 278 (Oxford University Press, 1956)

\section{Cambridge reviews}

SIR-You refer (Nature 339, 412; 1989) to "the independent review group set up to examine [Cambridge] University's management", but in fact the syndicate chaired by Sir Douglas Wass was appointed 'to consider the government of the University', a related but different question. The distinction is important, all the more so under a government that would like to see it disappear.

Gonville and Caius College,

A.W.F. EDWARDS 